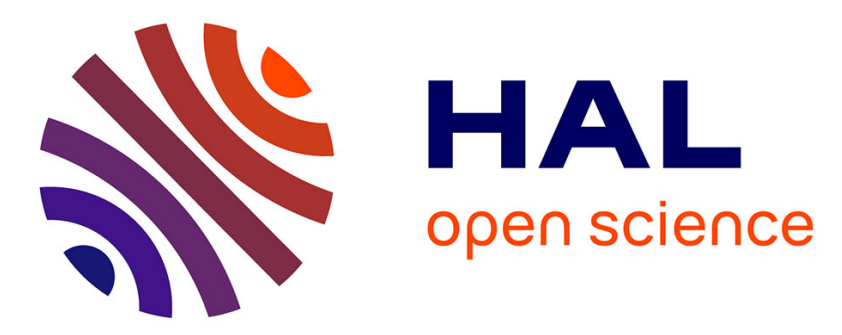

\title{
An existence proof for the stationary compressible Stokes problem
}

A Fettah, Thierry Gallouët, Lakehal H.

\section{To cite this version:}

A Fettah, Thierry Gallouët, Lakehal H.. An existence proof for the stationary compressible Stokes problem. Annales de la Faculté des Sciences de Toulouse. Mathématiques., 2014, 23, pp.847 - 875. 10.5802/afst.1427 . hal-01101959

\section{HAL Id: hal-01101959 \\ https://hal.science/hal-01101959}

Submitted on 12 Jan 2015

HAL is a multi-disciplinary open access archive for the deposit and dissemination of scientific research documents, whether they are published or not. The documents may come from teaching and research institutions in France or abroad, or from public or private research centers.
L'archive ouverte pluridisciplinaire HAL, est destinée au dépôt et à la diffusion de documents scientifiques de niveau recherche, publiés ou non, émanant des établissements d'enseignement et de recherche français ou étrangers, des laboratoires publics ou privés. 


\title{
AN EXISTENCE PROOF FOR THE STATIONARY COMPRESSIBLE STOKES PROBLEM
}

\author{
A. FETTAH, T. GALLOUËT, AND H. LAKEHAL
}

\begin{abstract}
In this paper, we prove the existence of a solution for a quite general stationary compressible Stokes problem including, in particular, gravity effects. The Equation Of State gives the pressure as an increasing superlinear function of the density. This existence result is obtained by passing to the limit on the solution of a viscous approximation of the continuity equation.
\end{abstract}

RÉsumÉ. dans cet article, nous prouvons l'existence d'une solution pour le problème de Stokes compressible stationnaire en tenant compte, en particulier, des effets gravitaires. L'équation d'état donne la pression comme une fonction strictement croissante superlinéaire de la densité. L'existence de solution est obtenue en passant à la limite sur une approximation visqueuse de l'équation de continuité.

\section{INTRODUCTION}

We consider the following problem:

$$
\begin{array}{r}
-\Delta u+\nabla p=f(x, \rho) \text { in } \Omega, \\
u=0 \text { on } \partial \Omega, \\
\operatorname{div}(\varphi(\rho) u)=0 \text { in } \Omega, \\
\rho \geq 0 \text { in } \Omega, \\
\int_{\Omega} \rho d x=M, \\
p=\eta(\rho) \text { in } \Omega .
\end{array}
$$

This problem is classical in fluid mechanics. In this case, $u$ is a vector valued function and stands for the velocity of the fluid. The functions $p$ and $\rho$ stand for the pressure and the density of the fluid (then $\rho \geq 0$ is necessary). Equation (1.1a) is the momentum equation, it is completed with the natural Dirichlet boundary condition (1.1b). The function $f$ represent a forcing term, including gravity effect, a main example is $f(x, \rho)=\tilde{f}(x)+g \rho$, where $g$ is the gravity constant. Equation (1.1c) is generally written with $\varphi(\rho)=\rho$ and corresponds to the mass conservation (or continuity equation). The total mass of the fluid is given by (1.1e). Finally (1.1f) is the Equation Of State (generally denoted as EOS). A main example is $\eta(\rho)=\rho^{\gamma}$ with $\gamma=5 / 3$ or $7 / 5$, or, more generally, $\gamma>1$ (the case $\gamma=1$ has also some interest and can be treated if $f$ does not depend on $\rho$, see for instance [11], in

2000 Mathematics Subject Classification. 35Q30,65N12,65N30,76M25.

Key words and phrases. Compressible Stokes equations, existence of solution. 
this case it is simpler). The Stokes equations and, more generally, the Navier-Stokes equation, in the evolution case and in the steady state case, are extensively used for a long time as models for fluid mechanics in the incompressible case (that is the case where $\rho$ is a constant) and in the compressible case. For the compressible case, a very well known paper is, for instance, [12], see also [19] or [1]. For the mathematical point de view, the existence of a weak solution for the incompressible evolution Navier-Stokes equations is due to J. Leray in the pioneering paper [15]. In the compressible case, the first result is due to P. L. Lions [16] (for $p=\rho^{\gamma}$, $\gamma>9 / 5)$.

Notations: For $a, b \in \mathbb{R}^{N}, a \cdot b$ denotes the usual scalar product of $a$ and $b$ in $\mathbb{R}^{N}$ and $|a|^{2}=a \cdot a$. For $a=\left(a_{1}, \ldots, a_{N}\right)$ and $b=\left(b_{1}, \ldots, b_{N}\right)$ with $a_{i}, b_{i} \in \mathbb{R}^{N}$ (for $i \in\{1, \ldots, N\})$, we set $a: b=\sum_{i=1}^{N} a_{i} \cdot b_{i}$ and $|a|^{2}=a: a$.

The set $\Omega$ is a connected bounded open set of $\mathbb{R}^{N}, N=2$ or 3 , with a Lipschitz continuous boundary. The real $M$ is positive. The function $f: \Omega \times \mathbb{R} \rightarrow \mathbb{R}^{N}$ satisfies the following hypothesis:

$$
\left\{\begin{array}{l}
f(x, s) \text { is measurable with respect to } x \in \Omega \text { for all } s \in \mathbb{R} \\
\text { and is continuous with respect to } s \in \mathbb{R} \text { for a.e. } x \in \Omega . \\
\text { There exists } B>0 \text { and } H \in L^{2}(\Omega) \text { such that, } \\
\text { for a.e. } x \in \Omega \text { and all } s \in \mathbb{R},|f(x, s)| \leq B(H(x)+|s|) .
\end{array}\right.
$$

The first part of Condition (1.2) (before the bound on $f$ ) is also called the Caratheodory condition and a function $f(x, s)$ satisfying this condition is usually called a Caratheodory function.

The function $\eta$ satisfy:

$$
\begin{aligned}
& \eta \in C\left(\mathbb{R}_{+}, \mathbb{R}_{+}\right), \eta(0)=0, \eta \text { is increasing, } \\
& \liminf _{s \rightarrow+\infty} \eta(s) / s=+\infty .
\end{aligned}
$$

The function $\varphi$ is an increasing Lipschitz continuous function from $\mathbb{R}$ to $\mathbb{R}$ and $\varphi(0)=0$. Then, there exists a constant $L>0$ such that

$$
\begin{aligned}
& \varphi \text { is increasing, } \\
& \varphi(0)=0 \text { and, for all } s_{1}, s_{2} \in \mathbb{R},\left|\varphi\left(s_{1}\right)-\varphi\left(s_{2}\right)\right| \leq L\left|s_{1}-s_{2}\right| .
\end{aligned}
$$

Remark 1.1. The hypothesis $\eta(0)=0$ is not a restriction since $p$ can be replaced by $(p-\eta(0))$ in the momemtum equation (Equation (1.1a)), and the EOS (Equation (1.1f)) can be written as $p-\eta(0)=\eta(\rho)-\eta(0)$. On the contrary, the hypothesis $\varphi(0)=0$ is important. In fact, this hypothesis allow us to prove that any solution $\rho$ (for $u$ given) of a regularized version (by adding some viscosity) of the mass equation (1.1c) has a constant sign (and then is positive if (1.1e) is satisfied with $M>0)$. This is related to the fact that $\rho=0$ is solution of (1.1c) which is not true if $\varphi(0) \neq 0$ (since, in general $u$ is not divergence free), see Section 2. Passing to the limit as the added viscosity tends to 0 leads to a nonnegative weak solution $\rho$ of (1.1c) (and (1.1d) is true).

Definition 1.2. Let $M>0$ and $\Omega$ be a connected bounded open set of $\mathbb{R}^{N}(N=2$ or 3) with a Lipschitz continuous boundary. Assume that (1.2), (1.3) and (1.4) 
are fulfilled. A weak solution of Problem (1.1) is a function $(u, p, \rho) \in \mathrm{H}_{0}^{1}(\Omega)^{N} \times$ $\mathrm{L}^{2}(\Omega) \times \mathrm{L}^{2}(\Omega)$ satisfying:

$$
\int_{\Omega} \boldsymbol{\nabla} u: \nabla v d x-\int_{\Omega} p \operatorname{div}(v) d x=\int_{\Omega} \boldsymbol{f}(x, \rho) \cdot v d x \text { for all } v \in \mathrm{H}_{0}^{1}(\Omega)^{N},
$$

$$
\int \varphi(\rho) u \cdot \nabla \psi d x=0 \text { for all } \psi \in W^{1, \infty}(\Omega)^{N},
$$

$$
\rho \geq 0 \text { a.e. in } \Omega, \int_{\Omega} \rho d x=M, p=\eta(\rho) \text { a.e. in } \Omega \text {. }
$$

The main objective of this paper is to prove the existence of a weak solution of Problem (1.1) (in the sense of Definition 1.2), namely the following theorem:

Theorem 1.3. Let $M>0$ and $\Omega$ be a connected bounded open set of $\mathbb{R}^{N}(N \geq$ 1) with a Lipschitz continuous boundary. Assume that (1.2), (1.3) and (1.4) are fulfilled. Then (1.5) has at least one solution.

The proof of Theorem 1.3 mainly uses the tools recently developed for the Navier Stokes in the books [16], [6] and [18]. It will be obtain by passing to the limit on the solution of a regularized problem (the existence of the solution of the regularized problem is also proved). In particular, the idea to add a viscosity to the continuity equation in order to obtain an approximate solution is already in the book of $\mathrm{P}$. L. Lions [16]. On the contrary, it seems to us that the way for proving the strong convergence of the pressure and the density by proving (4.22) (where we pass to the limit on the product of two weak convergences) is quite original and does not use the renormalized continuity equation (even if it is implicitly hidden in the proof), a tool in the theory of P. L. Lions. Indeed, the main interest of the (quite simple) proof given in this paper is probably that it can be adapted in order to prove the same result but by passing to the limit on the solution given by a numerical scheme (in particular using numerical schemes used in an industrial context). Such an existence proof of a weak solution of Problem (1.1) (by passing to the limit on the solution given by a numerical scheme) is done, for instance, in [7] for the particular case $f(x, \rho)=\bar{f}(x)+g(x) \rho(x)$, with $\bar{f} \in L^{2}(\Omega)$ and $g \in L^{\infty}(\Omega)$, and $\varphi(\rho)=\rho$. In this particular case $(f(x, \rho)=\bar{f}(x)+g(x) \rho(x)$ and $\varphi(\rho)=\rho)$, the hypothesis " $\eta$ is increasing" can be replaced by the weaker hypothesis " $\eta$ is nondecreasing" and Theorem 1.3 is still true (see Theorem 4.2). Since, in general, no uniqueness result is available for the weak solution of Problem (1.1), the convergence of a sequence of approximate solutions is obtained only up to a subsequence. Recent works were developed for the compressible stationary Navier-Stokes equations with $\eta(\rho)=\rho^{\gamma}$. The first papers [3] and [17] are devoted to the case $\gamma>3 / 2$ with a periodic boundary condition. The papers [9] [8], [20] deal with $\gamma>4 / 3$ and [14] deals with $\gamma>1$, also in the case of a periodic boundary condition. The paper [13] is devoted to the case $\gamma=1$ and another boundary condition, namely the Navier condition.

This paper is organized as follows. In Section 2, we present an existence and uniqueness result to a convection-diffusion equation with the Neumann boundary condition. This existence and uniqueness result is a main tool for proving, in Section 3 , the existence of a weak solution for the Problem (1.1) regularized by adding a viscous term in the mass conservation (equation (1.1c)) and with a truncation of $f$ and $\eta$. Then, in Section 4, we prove Therorem 1.3 (namely the existence of 
a solution to (1.5)) passing to the limit on a sequence of approximate solutions. Finally, in Section 5, we collect some useful lemmas.

\section{Convection-Diffusion with the Neumann Boundary condition}

In this section we consider here the following equation

$$
-\Delta \rho+\operatorname{div}(\varphi(\rho) u)=0 \text { in } \Omega,
$$

with the natural boundary condition which reads, if $\Omega, u$ and $\rho$ are regular enough, $-\nabla \rho \cdot n+\varphi(\rho) u \cdot n=0$ (where $n$ is the exterior normal vector to the boundary of $\Omega)$.

Under the hypothesis $u \in L^{p}(\Omega)^{N}$ for some $p>N$, the weak formulation of this problem is

$$
\left\{\begin{array}{l}
\rho \in H^{1}(\Omega) \\
\int_{\Omega} \nabla \rho(x) \cdot \nabla v(x) d x-\int_{\Omega} \varphi(\rho(x)) u(x) \cdot \nabla v(x) d x=0 \text { for all } v \in H^{1}(\Omega) .
\end{array}\right.
$$

The weak formulation (2.2) is meaningfull, at least if $\Omega$ is a bounded open set with a Lipschitz continuous boundary. Indeed, for $N>1$ (the case $N=1$ is easier), for $\rho \in H^{1}(\Omega)$, one has $\rho \in L^{q}(\Omega)$ for all $q<2 N /(N-2)$ (and even $q=2 N /(N-2)$ if $N>2$ ) and then, thanks to (1.4b), $\varphi(\rho) \in L^{q}(\Omega)$, for the same values of $q$. Then, for $u \in L^{p}(\Omega)^{N}$ and $p>N$, one has $\varphi(\rho) u \in L^{2}(\Omega)^{N}$ and $\varphi(\rho) u \cdot \nabla v \in L^{1}(\Omega)$ if $v \in H^{1}(\Omega)$.

We give in Theorem 2.1 an existence and uniqueness result for (2.2) along with some useful properties. A similar result (in the linear case, $\varphi(\rho)=\rho$ ) is given in $[4]$.

Theorem 2.1. Let $\Omega$ be a connected bounded open set of $\mathbb{R}^{N}(N=2$ or 3$)$ with a Lipschitz continuous boundary. Let $p>N, u \in L^{p}(\Omega)^{N}, M \geq 0$ and $\varphi$ be a function from $\mathbb{R}$ to $\mathbb{R}$ satisfying (1.4b). Then, there exist a unique solution to problem (2.2) satisfying the additional condition $\int_{\Omega} \rho(x) d x=M$. Furthermore one has the two following properties:

(1) $\rho>0$ a.e. on $\Omega$ if $M>0$ (and $\rho=0$ a.e. on $\Omega$ if $M=0$ ).

(2) For any $A>0$, there exists $C$ only depending on $A, p, M, \varphi$ and $\Omega$ such that, if $\rho$ is the solution of (2.2) with $\int_{\Omega} \rho(x) d x=M$, one has

$$
\||u|\|_{L^{p}(\Omega)} \leq A \Rightarrow\|\rho\|_{H^{1}(\Omega)} \leq C .
$$

Remark 2.2. Before giving the proof, we remark that the hypothesis " $\varphi$ increasing" (Hypothesis (1.4a)) is unuseful in Theorem 2.1. On the contrary, the hypothesis $\varphi(0)=0$ is crucial (in order to have the positivity of $\rho$ ).

\section{Proof}

The proof is divided in 3 steps.

Step 1 proves the a priori positivity of $\rho$. Namely, if $\rho$ satisfy $(2.2)$ with $\int_{\Omega} \rho(x) d x=$ $M$, then $\rho>0$ a.e. in $\Omega$ if $M>0$ and $\rho=0$ a.e. in $\Omega$ if $M=0$. Using similar arguments, we prove in this step the uniqueness (but not the existence) for all $M$ given, of the solution of (2.2) with $\int_{\Omega} \rho(x) d x=M$. Step 2 gives an a priori estimate on the solutions of (2.2) with $\int_{\Omega} \rho(x) d x=M$. Indeed, it gives the second property of Theorem 2.1 (but not yet the existence result). Step 3 gives the desired existence result, using the Leray-Schauder topological degree.

Step 1, a priori positivity and uniqueness 
Let $\rho$ be a solution of $(2.2)$ with $\int_{\Omega} \rho(x) d x=M$. In order to prove that $\rho>0$ a.e. if $M>0$, we argue by contradiction. We set $\omega=\{\rho \leq 0\}$ and we assume that $\lambda_{N}(\omega)>0$ (where $\lambda_{N}$ is the Lebesgue measure on $\mathbb{R}^{N}$ ).

For $n \in \mathbb{N}^{*}$ we define $T_{n}$ from $\mathbb{R}$ to $\mathbb{R}$ by $T_{n}(s)=\min \left\{\frac{1}{n}, \max \{s, 0\}\right\}$. It is well known that the function $T_{n}(\rho)$ belongs to $H^{1}(\Omega)$ and that

$$
\nabla T_{n}(\rho)=1_{0<\rho<\frac{1}{n}} \nabla \rho \text { a.e. in } \Omega .
$$

Then, taking $v=T_{n}(\rho)$ in (2.2) leads to

$$
\int_{\Omega}\left|\nabla T_{n}(\rho)\right|^{2} d x=\int_{\Omega} \varphi(\rho) u \cdot \nabla T_{n}(\rho) d x \leq L \frac{a_{n}}{n}\left(\int_{\Omega}\left|\nabla T_{n}(\rho)\right|^{2} d x\right)^{\frac{1}{2}},
$$

with

$$
a_{n}=\left(\int_{0<\rho<\frac{1}{n}}|u|^{2} d x\right)^{\frac{1}{2}} .
$$

Since $u \in L^{2}(\Omega)^{N}$ and $\lim _{n \rightarrow \infty} \lambda_{N}\left(\left\{0<\rho<\frac{1}{n}\right\}\right)=0$, one has $\lim _{n \rightarrow \infty} a_{n}=0$. Using the fact that $\|z\|_{L^{1}(\Omega)} \leq\|z\|_{L^{2}(\Omega)} \lambda_{N}(\Omega)^{1 / 2}$, we have

$$
\left\|\left|\nabla T_{n}(\rho)\right|\right\|_{L^{1}(\Omega)} \leq\left\|\left|\nabla T_{n}(\rho)\right|\right\|_{L^{2}(\Omega)} \lambda_{N}(\Omega)^{1 / 2} \leq L \frac{a_{n}}{n} \lambda_{N}(\Omega)^{1 / 2} .
$$

We now remark that $T_{n}(\rho)=0$ a.e. on $\omega$. Since $\lambda_{N}(\omega)>0$, Lemma 5.1 (which uses the connexity of $\Omega$ ) gives the existence of $C$, only depending on $\Omega$ and $\omega$ such that

Since

$$
\left\|T_{n}(\rho)\right\|_{L^{1}(\Omega)} \leq C\left\|\left|\nabla T_{n}(\rho)\right|\right\|_{L^{1}(\Omega)} .
$$

we then have

$$
\left\|T_{n}(\rho)\right\|_{L^{1}(\Omega)} \geq \frac{1}{n} \lambda_{N}\left(\left\{\rho \geq \frac{1}{n}\right\}\right),
$$

$$
\lambda_{N}\left(\left\{\rho \geq \frac{1}{n}\right\}\right) \leq L C a_{n} \lambda_{N}(\Omega)^{1 / 2} .
$$

Passing to the limit as $n \rightarrow \infty$ leads to $\lambda_{N}(\{\rho>0\})=0$, that is $\rho \leq 0$ a.e..

If $M>0$, it is impossible since $\int_{\Omega} \rho d x=M>0$. Then, we conclude that $\lambda_{N}(\omega)=$ 0 , which gives $\rho>0$ a.e. in $\Omega$.

If $M=0$, one has $\int_{\Omega} \rho d x=M=0$ and then from $\rho \leq 0$ a.e. we conlude that $\rho=0$ a.e. in $\Omega$.

By a similar argument, we now prove the uniqueness of the solution of (2.2) with $\int_{\Omega} \rho d x=M$. Let $\rho_{1}$ and $\rho_{2}$ be two solutions of $(2.2)$ with $\int_{\Omega} \rho_{1} d x=\int_{\Omega} \rho_{2} d x=M$. We set $\rho=\rho_{1}-\rho_{2}$. Taking the difference of the equations satisfied by $\rho_{1}$ and $\rho_{2}$, with $v=T_{n}(\rho)$ as test function, we obtain (2.3) with (2.4). Since $\int_{\Omega} \rho(x) d x=0$, we conclude (as in the preceding proof) that $\rho=0$ a.e.. This gives the uniqueness of the solution of (2.2) with $\int_{\Omega} \rho d x=M$.

Actually, it is interesting to notice that the present step consists essentially to prove that any solution of (2.2) has a constant sign.

Step 2, a priori estimate

Let $A>0$ and assume that $\||u|\|_{L^{p}(\Omega)} \leq A$. Let $\rho$ be a solution of (2.2) with $\int_{\Omega} \rho d x=M$. Taking $v=\rho$ in (2.2) and using Hölder Inequality with $q=\frac{2 p}{p-2}$ (which gives $\frac{1}{p}+\frac{1}{q}=\frac{1}{2}$ ) leads to

$$
\||\nabla \rho|\|_{L^{2}(\Omega)}^{2}=\int_{\Omega}|\nabla \rho|^{2} d x \leq L\||u|\|_{L^{p}(\Omega)}\|\rho\|_{L^{q}(\Omega)}\||\nabla \rho|\|_{L^{2}(\Omega)} .
$$


We choose $\bar{q}$ such that $q<\bar{q}<+\infty$ if $N=2$ and $\bar{q}=6$ if $N=3$ (which gives $q<\bar{q}$ ). By Sobolev Inequality, there exists $C_{s}>0$ only depending on $\Omega$ such that

$$
\|\rho\|_{L^{\bar{q}}(\Omega)} \leq C_{s}\|\rho\|_{H^{1}(\Omega)} .
$$

By Hölder Inequality, we also have, with $\theta=\frac{\bar{q}-q}{q(\bar{q}-1)} \in(0,1)$ (which only depends on $p$ and $N$ ),

This gives

$$
\|\rho\|_{L^{q}(\Omega)} \leq\|\rho\|_{L^{1}(\Omega)}^{\theta}\|\rho\|_{L^{\bar{q}}(\Omega)}^{1-\theta} .
$$

and, with $(2.5)$,

$$
\|\rho\|_{L^{q}(\Omega)} \leq M^{\theta} C_{s}^{1-\theta}\|\rho\|_{H^{1}(\Omega)}^{1-\theta}
$$

We now use the Poincaré-Wirtinger Inequality. It gives the existence of $C_{p}>0$ only depending on $\Omega$ such that

$$
\left\|\rho-M \lambda_{N}(\Omega)^{-1}\right\|_{L^{2}(\Omega)} \leq C_{p}\||\nabla \rho|\|_{L^{2}(\Omega)}
$$

(the connexity of $\Omega$ is used once again here).

Then we have

$$
\|\rho\|_{L^{2}(\Omega)} \leq\left\|\rho-M \lambda_{d}(\Omega)^{-1}\right\|_{L^{2}(\Omega)}+M \lambda_{d}(\Omega)^{-\frac{1}{2}} \leq C_{p}\||\nabla \rho|\|_{L^{2}(\Omega)}+M \lambda_{d}(\Omega)^{-\frac{1}{2}} .
$$

This gives

$$
\|\rho\|_{H^{1}(\Omega)} \leq\left(C_{p}+1\right)\||\nabla \rho|\|_{L^{2}(\Omega)}+M \lambda_{d}(\Omega)^{-\frac{1}{2}} .
$$

Finally, with (2.6) and (2.7), we obtain the existence of $C_{1}$ and $C_{2}$ only depending on $A, M, p, L$, and $\Omega$ such that

$$
\|\rho\|_{H^{1}(\Omega)} \leq C_{1}\|\rho\|_{H^{1}(\Omega)}^{1-\theta}+C_{2} .
$$

Since $\theta>0$, this gives the existence of $C$ only depending on $A, M, p, L$ and $\Omega$ such that $\|\rho\|_{H^{1}(\Omega)} \leq C$ and concludes this step.

Step 3, existence

For $u$ and $M$ given, we have to prove the existence of a solution to (2.2) with $\int_{\Omega} \rho d x=M$. Let $t \in[0,1]$ and $q=\frac{2 p}{p-2}$. We now define a continuous and compact application from $[0,1] \times L^{q}(\Omega)$ in $L^{q}(\Omega)$. For $t \in[0,1]$ and $\bar{\rho} \in L^{q}(\Omega)$, since $u \varphi(\bar{\rho}) \in L^{2}(\Omega)^{N}$, it is well known that there exists a unique weak solution of the following problem (which is the classical Neumann problem):

$$
\begin{aligned}
& \rho \in H^{1}(\Omega), \int_{\Omega} \rho d x=0, \\
& \int_{\Omega} \nabla \rho \cdot \nabla v(x) d x=t \int_{\Omega} u \varphi(\bar{\rho}) \cdot \nabla v(x) d x \text { for all } v \in H^{1}(\Omega) .
\end{aligned}
$$

Since $H^{1}(\Omega) \subset L^{q}(\Omega)$, we can define the application $F$ from $[0,1] \times L^{q}(\Omega)$ in $L^{q}(\Omega)$ by setting

$$
F(t, \bar{\rho})=t\left(\rho+\frac{M}{\lambda_{N}(\Omega)}\right)
$$

One has $\int_{\Omega} F(t, \bar{\rho}) d x=t M$. Then, if $\rho=F(1, \rho)$, the function $\rho$ is a solution of (2.2) with $\int_{\Omega} \rho d x=M$. We prove below the existence of such a function $\rho$ using the invariance by homotopy of the Leray-Schauder topological degree.

We first prove that $F$ is continuous. Let $\left(t_{n}, \bar{\rho}_{n}\right)_{n \in \mathbb{N}}$ be a sequence of $[0,1] \times L^{q}(\Omega)$ such that $t_{n} \rightarrow t\left(\right.$ in $\mathbb{R}$ ) and $\bar{\rho}_{n} \rightarrow \bar{\rho}$ in $L^{q}(\Omega)$ as $n \rightarrow \infty$. Since $\varphi$ is Lipschitz 
continuous (hypothesis (1.4b)) one also has $\varphi\left(\bar{\rho}_{n}\right) \rightarrow \varphi(\bar{\rho})$ in $L^{q}(\Omega)$ as $n \rightarrow \infty$ (the continuity in $L^{q}$ of the application $\rho \mapsto \varphi(\rho)$, when $\varphi$ is Lipschitz continuous is well known, see, for instance, [10]). Let $\rho_{n}$ be the solution of $(2.8)$ with $t_{n}$ and $\bar{\rho}_{n}$ instead of $t$ and $\bar{\rho}$. The sequence $\left(\rho_{n}\right)_{n \in \mathbb{N}}$ is bounded in $H^{1}(\Omega)$. Then, up a subsequence, there exists $\rho \in H^{1}(\Omega)$ such that $\rho_{n} \rightarrow \rho$ weakly in $H^{1}(\Omega)$. Then, passing to the limit (as $n \rightarrow \infty$ ) in the equations satisfied by $\rho_{n}$, we prove that $\rho$ is solution of (2.8). Since this solution is unique, we obtain that $\rho_{n} \rightarrow \rho$ weakly in

$H^{1}(\Omega)$ without extraction of a sequence. Indeed, it is even possible to prove that $\rho_{n} \rightarrow \rho$ in $H^{1}(\Omega)$ since taking $u_{n}$ as test function in the equation satisfied by $u_{n}$ one obtains $\lim _{n \rightarrow \infty}\left\|\left|\nabla u_{n}\right|\right\|_{L^{2}(\Omega)}=\||\nabla u|\|_{L^{2}(\Omega)}$. Finally, since the space $H^{1}(\Omega)$ is continuously embedded in $L^{q}(\Omega)$ (this is the Sobolev Embedding Theorem since $q<2 N /(N-2))$, one has $\rho_{n} \rightarrow \rho$ in $L^{q}(\Omega)$ and this proves that $F$ is continuous from $[0,1] \times L^{q}(\Omega)$ to $L^{q}(\Omega)$.

Furthermore, since $H^{1}(\Omega)$ is compactly embedded in $L^{q}(\Omega)$ (once again since $q<$ $2 N /(N-2))$, the function $F$ is compact from $[0,1] \times L^{q}(\Omega)$ to $L^{q}(\Omega)$.

Now, we remark that

$$
\left(t \in[0,1], \rho \in L^{q}(\Omega), \rho=F(t, \rho)\right) \Rightarrow \rho \text { is solution of }(2.2) \text { with } \int_{\Omega} \rho=t M
$$

A quick look on Step 2 gives an $H^{1}$ estimate on $\rho$, namely,

$$
\left(t \in[0,1], \rho \in L^{q}(\Omega), \rho=F(t, \rho)\right) \Rightarrow \exists C>0 \text { such that }\|\rho\|_{H^{1}(\Omega)} \leq C .
$$

Then, there exists $R>0$ such that

$$
\left(t \in[0,1], \rho \in L^{q}(\Omega), \rho=F(t, \rho)\right) \Rightarrow\|\rho\|_{L^{q}(\Omega)}<R .
$$

Let $B_{R}$ be the ball of radius $R$ and center 0 in $L^{q}(\Omega)$. The topological degree of $I d-F(t, \cdot)$ (where $I d$ is the application $\rho \mapsto \rho$ ) on $B_{R}$ associated to point 0 is well defined and is independant of $t \in[0,1]$. This gives $d\left(\operatorname{Id}-F(1, \cdot), B_{R}, 0\right)=$ $d\left(I d-F(0, \cdot), B_{R}, 0\right)$. But, since $F(0, \cdot)=0$, we have $d\left(I d-F(0, \cdot), B_{R}, 0\right)=1$. Then $d\left(I d-F(1, \cdot), B_{R}, 0\right)=1$. This proves the existence of $\rho \in B_{R}$ such that $\rho=F(1, \rho)$ and concludes the proof of the Theorem 2.1.

\section{The REGUlarized PROBLEM}

In this section, we prove the existence of a solution for Problem 1.1 regularized by adding a diffusion term in (1.1c) and replacing $f$ and $\eta$ by truncated functions.

For $n \in \mathbb{N}$, one defines the function $T_{n}$ from $\mathbb{R}$ to $\mathbb{R}$ by setting

$$
T_{n}(s)=\min \{\max \{s,-n\}, n\}
$$

For $s=\left(s_{1}, \ldots, s_{N}\right) \in \mathbb{R}^{N}$, one sets $T_{n}(s)=\left(T_{n}\left(s_{1}\right), \ldots, T_{n}\left(s_{N}\right)\right)$. Then, the regularized problem reads, for $n, l, m \in \mathbb{N}^{*}$, 


$$
\begin{aligned}
& u \in H_{0}^{1}(\Omega)^{N}, \rho \in H^{1}(\Omega), p \in L^{2}(\Omega) \\
& \int_{\Omega} \nabla u: \nabla v d x-\int_{\Omega} p \operatorname{div}(v) d x=\int_{\Omega} f_{l}(x, \rho) \cdot v d x, \forall v \in \mathrm{H}_{0}^{1}(\Omega)^{N}, \\
& \int_{\Omega} \varphi(\rho) u \cdot \nabla \psi d x-\frac{1}{n} \int_{\Omega} \nabla \rho(x) \cdot \nabla \psi(x) d x=0 \text { for all } \psi \in H^{1}(\Omega), \\
& \rho>0 \text { a.e. in } \Omega, \int_{\Omega} \rho d x=M, p=\eta_{m}(\rho) \text { a.e. in } \Omega,
\end{aligned}
$$

where $f_{l}(x, s)=T_{l}(f(x, s))$ and $\eta_{m}(s)=T_{m}(\eta(s))$ for $x \in \Omega$ and $s \in \mathbb{R}$.

Proposition 3.1. Let $M>0$ and $\Omega$ be a connected bounded open set of $\mathbb{R}^{N}$ $(N \geq 1)$ with a Lipschitz continuous boundary. Assume that (1.2), (1.3) and (1.4b) are fulfilled. Let $l, m, n \in \mathbb{N}^{*}$. Then (3.1) has at least one solution.

\section{Proof}

We will apply the Brouwer Fixed Point Theorem to a convenient application $T$ from $L^{2}(\Omega)$ to $L^{2}(\Omega)$.

Let $\tilde{\rho} \in L^{2}(\Omega)$. We set $\tilde{p}=\eta_{m}\left(\tilde{\rho}^{+}\right)$. Knowing $\tilde{p}$ and $\tilde{\rho}$, The classical Lax-Milgram lemma gives existence and uniqueness of $u$ solution of the following problem:

$$
\left\{\begin{array}{l}
u \in \mathrm{H}_{0}^{1}(\Omega)^{N}, \\
\int_{\Omega} \nabla u: \nabla v d x=\int_{\Omega} \tilde{p} \operatorname{div}(v) d x+\int_{\Omega} f_{l}(x, \tilde{\rho}) \cdot v d x, \forall v \in \mathrm{H}_{0}^{1}(\Omega)^{N} .
\end{array}\right.
$$

Knowing $u$, Therorem 2.1 gives existence and uniqueness of $\rho$ solution of the following problem:

$$
\left\{\begin{array}{l}
\rho \in H^{1}(\Omega), \int_{\Omega} \rho(x) d x=M \\
\frac{1}{n} \int_{\Omega} \nabla \rho(x) \cdot \nabla \psi(x) d x-\int_{\Omega} \varphi(\rho(x)) u(x) \cdot \nabla \varphi(x) d x=0, \forall \psi \in H^{1}(\Omega) .
\end{array}\right.
$$

Then, we set $T(\tilde{\rho})=\rho$. We now prove that the function $T$ is continuous and compact (from $L^{2}(\Omega)$ to $L^{2}(\Omega)$ ) and that there exists $R>0$ such that $\operatorname{Im}(T) \subset B_{R}$, where $B_{R}=\left\{\rho \in L^{2}(\Omega) ;\|\rho\|_{L^{2}(\Omega)} \leq R\right\}$ (and we conclude with the Brouwer theorem).

- Continuity of $T$

This is the tricky part of the proof of Proposition 3.1. Let $\left(\tilde{\rho_{k}}\right)_{k \in \mathbb{N}}$ a sequence in $L^{2}(\Omega)$. We suppose that $\tilde{\rho_{k}}$ converges to $\tilde{\rho}$ in $L^{2}(\Omega)$ and we will prove that $\rho_{k}=T\left(\tilde{\rho_{k}}\right) \rightarrow T(\tilde{\rho})$ in $L^{2}(\Omega)$ as $k \rightarrow+\infty$.

We first remark that, as $k \rightarrow+\infty, \tilde{\rho_{k}} \rightarrow \tilde{\rho}$ and $\tilde{p_{k}}=\eta_{m}\left({\tilde{\rho_{k}}}^{+}\right) \rightarrow \tilde{p}=\eta_{m}\left(\tilde{\rho}^{+}\right)$in $L^{2}(\Omega)$ and $f_{l}\left(\cdot, \tilde{\rho_{k}}\right) \rightarrow f_{l}(\cdot, \tilde{\rho})$ in $L^{2}(\Omega)$ (this can be proven arguing by contradiction and using the a.e. convergence or directly with the convergence in measure as in Theorem 4.45 in [10] for $\left.L^{1}\right)$. Let $u$ be the solution of (3.2) and, for $k \in \mathbb{N}, u_{k}$ be the solution of (3.2) with $\tilde{p_{k}}$ and $\tilde{\rho_{k}}$ in the right hand side. A classical result for an elliptic equation with Dirichlet boundary condition gives that $u_{k} \rightarrow u$ in $H_{0}^{1}(\Omega)$, as $k \rightarrow+\infty$. Furthermore, taking $v=u_{k}$ as test function in the equation satisfied by $u_{k}$, we obtain the existence of $C_{1}$ only depending on $m, l$ and $\Omega$ such that

$$
\left\|u_{k}\right\|_{H_{0}^{1}(\Omega)^{N}} \leq C_{1}
$$


Then, Sobolev embedding gives the existence of $C_{2}$ only depending on $m, l$ and $\Omega$ and $p$ for $p \in[1,+\infty)$ if $N=2$ and $p=6$ for $N=3$, such that, for all $k \in \mathbb{N}$,

$$
\left\|u_{k}\right\|_{L^{p}(\Omega)^{N}} \leq C_{2}
$$

Let now $\rho_{k}$ be the solution of (3.3) with $u=u_{k}$, namely the solution of the following problem:

$$
\begin{aligned}
& \rho_{k} \in H^{1}(\Omega), \int_{\Omega} \rho_{k}=M, \\
& \frac{1}{n} \int_{\Omega} \nabla \rho_{k}(x) \cdot \nabla v(x) d x-\int_{\Omega} \varphi\left(\rho_{k}(x)\right) u_{k}(x) \cdot \nabla v(x) d x=0, \forall v \in H^{1}(\Omega) .
\end{aligned}
$$

Theorem 2.1 and inequality (3.5) gives the existence of $C_{3}$ only depending on $n$, $m, l, \varphi, M$, and $\Omega$ such that

$$
\left\|\rho_{k}\right\|_{H^{1}(\Omega)} \leq C_{3}
$$

and then, there exists $\rho \in H^{1}(\Omega)$ such that, up to a subsequence,

$$
\rho_{k} \rightarrow \rho \text { in } L^{2}(\Omega) \text { and weakly in } H^{1}(\Omega) .
$$

Passing to the limit (as $k \rightarrow+\infty$ ) in (3.6) gives that $\rho$ is solution of (3.3). Thanks to the uniqueness of the solution of (3.3), this give (arguing by contradiction) that $\rho_{k} \rightarrow \rho$ (in $L^{2}(\Omega)$ and weakly in $H^{1}(\Omega)$ ), as $k \rightarrow \infty$, without extraction of a sequence. Since $\rho=T(\tilde{\rho})$, we have proven the continuity of $T$ from $L^{2}(\Omega)$ in $L^{2}(\Omega)$.

- Compactness of $T$ and existence of $R$.

This part is a consequence of the estimate (3.7). Actually, this estimate gives that $\operatorname{Im}(T)$ is bounded in $H^{1}(\Omega)$ and then is compact in $L^{2}(\Omega)$, thanks to Rellich Theorem. This gives the compactness of $T$ and the existence of $R>0$ such that $\operatorname{Im}(T) \subset B_{R}$. We then conclude, using the Brouwer Fixed Point Theorem, that there exists $\rho \in L^{2}(\Omega)$ such that $T(\rho)=\rho$ which gives that $\rho$ is a solution of problem (3.1) (in particular, we recall that Theorem 2.1 gives that $\rho>0$ a.e. in $\Omega$.

\section{Proof of Theorem 1.3}

In this section we pass to the limit on the regularized problem in order to prove the existence of a solution to (1.5). We assume that the hypotheses of Theorem 1.3 are satisfied. Thanks to the previous section, we know that the regularized problem has a solution. This regularized problem is defined with three parameters which are $m, l$, and $n$. We will first let $m \rightarrow+\infty$ (Step 1), then $l \rightarrow+\infty$ (Step 2) and finally $n \rightarrow+\infty$ (Step 3).

Step $1, m \rightarrow+\infty$ 
In this step $l$ and $n$ are fixed in $\mathbb{N}^{*}$ and we prove the existence of a solution to (3.1) with $\eta$ instead of $\eta_{m}$, namely a solution to (4.1).

$$
\begin{aligned}
& u \in H_{0}^{1}(\Omega)^{N}, \rho \in H^{1}(\Omega), p \in L^{2}(\Omega), \\
& \int_{\Omega} \nabla u: \nabla v d x-\int_{\Omega} p \operatorname{div}(v) d x=\int_{\Omega} f_{l}(x, \rho) \cdot v d x, \forall v \in \mathrm{H}_{0}^{1}(\Omega)^{N}, \\
& \int_{\Omega} \varphi(\rho) u \cdot \nabla \psi d x-\frac{1}{n} \int_{\Omega} \nabla \rho(x) \cdot \nabla \psi(x) d x=0 \text { for all } \psi \in H^{1}(\Omega), \\
& \rho>0 \text { a.e. in } \Omega, \int_{\Omega} \rho d x=M, p=\eta(\rho) \text { a.e. in } \Omega .
\end{aligned}
$$

Let $\left(u_{m}, p_{m}, \rho_{m}\right)$ be a solution of (3.1). Taking $u_{m}$ as test function in (3.1b) yields (where $\|u\|_{H_{0}^{1}(\Omega)^{N}}=\||\nabla u|\|_{L^{2}(\Omega)}$ )

$$
\left\|u_{m}\right\|_{H_{0}^{1}(\Omega)^{N}}^{2}-\int_{\Omega} p_{m} \operatorname{div}\left(u_{m}\right) d x=\int_{\Omega} f_{l}\left(x, \rho_{m}\right) \cdot u_{m} d x .
$$

which gives, since $p_{m}=\eta_{m}\left(\rho_{m}\right)$,

$$
\left\|u_{m}\right\|_{H_{0}^{1}(\Omega)^{N}}^{2}-\int_{\Omega} \eta_{m}\left(\rho_{m}\right) \operatorname{div}\left(u_{m}\right) d x=\int_{\Omega} f_{l}\left(x, \rho_{m}\right) \cdot u_{m} d x .
$$

We now use Lemma 5.2. It gives, thanks to the fact that $\left(\rho_{m}, u_{m}\right)$ satisfy Equation (3.1c), $\int_{\Omega} \eta_{m}\left(\rho_{m}\right) \operatorname{div}\left(u_{m}\right) d x \leq 0$ (this Lemma uses hypothesis (1.4b) on $\varphi$ and the fact that $\varphi(s)>0$ for $s>0)$. Then, we have

$$
\left\|u_{m}\right\|_{H_{0}^{1}(\Omega)^{N}}^{2} \leq \int_{\Omega} f_{l}\left(x, \rho_{m}\right) \cdot u_{m} d x .
$$

Using Poincaré and Hölder inequalities, we obtain the existence of $C_{1}$ only depending on $l$ and $\Omega$ such that

$$
\left\|u_{m}\right\|_{H_{0}^{1}(\Omega)^{N}} \leq C_{1} .
$$

Using (4.2) and theorem 2.1, there exists $C_{2}$ only depending on $l, \Omega, M, \varphi$ and $n$ such that

$$
\left\|\rho_{m}\right\|_{H^{1}(\Omega)} \leq C_{2} .
$$

In order to obtain a bound for $p_{m}$ in $\mathrm{L}^{2}(\Omega)$, we now choose $v$ given by Lemma 5.3 with $q=p_{m}-m\left(p_{m}\right)$, where $m\left(p_{m}\right)$ is the mean value of $p_{m}$. Taking $v$ in (3.1b) and using $\int_{\Omega} \operatorname{div}(v) d x=0$ give

$$
\int_{\Omega}\left(p_{m}-m\left(p_{m}\right)\right)^{2} d x=\int_{\Omega}\left(f_{l}\left(x, \rho_{m}\right) \cdot v-\nabla u_{m}: \nabla v\right) d x .
$$

Then, since $\|v\|_{H_{0}^{1}(\Omega)^{N}} \leq C_{d}\left\|p_{m}-m\left(p_{m}\right)\right\|_{\mathrm{L}^{2}(\Omega)}\left(C_{d}\right.$ given by Lemma 5.3) and $\left\|u_{m}\right\|_{H_{0}^{1}(\Omega)^{N}} \leq C_{1}$, the preceding inequality leads to an estimate on the $L^{2}$-norm of $\left(p_{m}-m\left(p_{m}\right)\right)$, i.e. the existence of $C_{3}$, only depending on $\Omega$ and $l$, such that

$$
\left\|p_{m}-m\left(p_{m}\right)\right\|_{L^{2}(\Omega)} \leq C_{3} .
$$

We now use the fact that $\int_{\Omega} \rho_{m} d x=M$ to deduce an estimate on $\left\|p_{m}\right\|_{L^{2}(\Omega)}$. The function $\eta$ is a one-to-one function from $\mathbb{R}_{+}$onto $\mathbb{R}_{+}$. We denote by $\bar{\eta}$ its reciprocal function (that is $\bar{\eta}(\eta(s))=\eta(\bar{\eta}(s))=s$ for all $s \in \mathbb{R}_{+}$). we thus get

$$
\int_{\Omega} \bar{\eta}\left(p_{m}\right) d x=\int_{\Omega} \bar{\eta}\left(\eta_{m}\left(\rho_{m}\right)\right) d x \leq \int_{\Omega} \bar{\eta}\left(\eta\left(\rho_{m}\right)\right) d x=\int_{\Omega} \rho_{m} d x=M .
$$


and then, using (4.4) and Lemma 5.4, there exists $C_{4}$, only depending on the $\Omega, l$, $\eta$ and $M$, such that:

$$
\left\|p_{m}\right\|_{\mathrm{L}^{2}(\Omega)} \leq C_{4} .
$$

Remark 4.1. The fact that $\eta$ is increasing is not necessary here. If $\eta$ is only nondecreasing, the estimate on $p$ is obtained using the reciprocal function of $s \mapsto \eta(s)+s$ (instead of the reciprocal function of $\eta$ ). This is useful for Theorem 4.2.

Thanks to the estimates (4.2), (4.3) and (4.5), we have, up to a subsequence, as $m \rightarrow+\infty$,

$$
\begin{aligned}
u_{m} & \rightarrow u \text { weakly in } H_{0}^{1}(\Omega)^{N}, \\
\rho_{m} \rightarrow \rho \text { a.e., } & \text { in } L^{2}(\Omega) \text { and weakly in } H^{1}(\Omega), \\
p_{m} & \rightarrow p \text { weakly in } L^{2}(\Omega) .
\end{aligned}
$$

Then, passing to the limit (as $m \rightarrow+\infty$ ) in the equations satisfied by $\left(u_{m}, \rho_{m}, p_{m}\right)$ gives that $(u, p, \rho)$ is solution of (4.1). In particular, since $\eta_{m}\left(\rho_{m}\right) \rightarrow \eta(\rho)$ a.e and $\left(\eta_{m}\left(\rho_{m}\right)\right)_{m \in \mathbb{N}}$ is bounded in $L^{2}(\Omega)$, one has $\eta_{m}\left(\rho_{m}\right) \rightarrow \eta(\rho)$ in $L^{q}(\Omega)$ for all $q<2$. Since $\eta_{m}\left(\rho_{m}\right)=p_{m} \rightarrow p$ weakly in $L^{2}(\Omega)$, we then conclude that $p=\eta(\rho)$. We recall also that the fact that $\rho>0$ a.e. in $\Omega$ is given by Theorem 2.1. This concludes the proof of Step 1.

Step 2, $l \rightarrow+\infty$

In this step $n$ is fixed in $\mathbb{N}^{*}$ and we prove the existence of a solution to (4.1) with $f$ instead of $f_{l}$, namely a solution to (4.6).

$$
\begin{aligned}
& u \in H_{0}^{1}(\Omega)^{N}, \rho \in H^{1}(\Omega), p \in L^{2}(\Omega), \\
& \int_{\Omega} \nabla u: \nabla v d x-\int_{\Omega} p \operatorname{div}(v) d x=\int_{\Omega} f(x, \rho) \cdot v d x, \forall v \in \mathrm{H}_{0}^{1}(\Omega)^{N}, \\
& \int_{\Omega} \varphi(\rho) u \cdot \nabla \psi d x-\frac{1}{n} \int_{\Omega} \nabla \rho(x) \cdot \nabla \psi(x) d x=0 \text { for all } \psi \in H^{1}(\Omega), \\
& \rho>0 \text { a.e. in } \Omega, \int_{\Omega} \rho d x=M, p=\eta(\rho) \text { a.e. in } \Omega .
\end{aligned}
$$

Let $\left(u_{l}, p_{l}, \rho_{l}\right)$ be a solution of (4.1). The main additionnal difficulty with respect to Step 1 is to obtain the $H_{0}^{1}(\Omega)$ estimate on $u_{l}$. Taking $u_{l}$ as test function in $(4.1 \mathrm{~b})$ yields

$$
\left\|u_{l}\right\|_{H_{0}^{1}(\Omega)^{N}}^{2}-\int_{\Omega} p_{l} \operatorname{div}\left(u_{l}\right) d x=\int_{\Omega} f_{l}\left(x, \rho_{l}\right) \cdot u_{l} d x .
$$

which gives, since $p_{l}=\eta\left(\rho_{l}\right)$,

$$
\left\|u_{l}\right\|_{H_{0}^{1}(\Omega)^{N}}^{2}-\int_{\Omega} \eta\left(\rho_{l}\right) \operatorname{div}\left(u_{l}\right) d x=\int_{\Omega} f_{l}\left(x, \rho_{l}\right) \cdot u_{l} d x .
$$

Thanks to the fact that $\left(\rho_{l}, u_{l}\right)$ satisfy Equation (4.1c) and using Lemma 5.2 one has $\int_{\Omega} \eta\left(\rho_{l}\right) \operatorname{div}\left(u_{l}\right) d x \leq 0$. Then, we have

$$
\left\|u_{l}\right\|_{H_{0}^{1}(\Omega)^{N}}^{2} \leq \int_{\Omega} f_{l}\left(x, \rho_{l}\right) \cdot u_{l} d x .
$$

Using Poincaré and Cauchy-Schwarz inequalities, we obtain the existence of $C_{1}$ only depending on $f$ and $\Omega$ such that

$$
\left\|u_{l}\right\|_{H_{0}^{1}(\Omega)^{N}} \leq C_{1}\left(\left\|\rho_{l}\right\|_{L^{2}(\Omega)}+1\right) .
$$


Using (1.3b) and $p_{l}=\eta\left(\rho_{l}\right)$, for all $\epsilon>0$ there exists $C_{\epsilon}$ only depending on $\epsilon, \eta$ and $\Omega$ such that:

$$
\left\|\rho_{l}\right\|_{L^{2}(\Omega)} \leq C_{\epsilon}+\epsilon\left\|p_{l}\right\|_{L^{2}(\Omega)} .
$$

Then, with (4.7), for all $\epsilon>0$, there exists $\widetilde{C_{\epsilon}}$, only depending on $\epsilon, f$, and $\Omega$, such that

$$
\left\|u_{l}\right\|_{H_{0}^{1}(\Omega)^{N}} \leq \widetilde{C_{\epsilon}}+\epsilon\left\|p_{l}\right\|_{L^{2}(\Omega)} .
$$

We now choose (as in Step 1) $v$ given by Lemma 5.3 with $q=p_{l}-m\left(p_{l}\right)$, where $m\left(p_{l}\right)$ is the mean value of $p_{l}$. Taking $v$ in (4.1b) and using $\int_{\Omega} \operatorname{div}(v) d x=0$ give

$$
\int_{\Omega}\left(p_{l}-m\left(p_{l}\right)\right)^{2} d x=\int_{\Omega}\left(f_{l}\left(x, \rho_{l}\right) \cdot v-\nabla u_{l}: \nabla v\right) d x .
$$

Since $\|v\|_{H_{0}^{1}(\Omega)^{N}} \leq C_{d}\left\|p_{m}-m\left(p_{m}\right)\right\|_{\mathrm{L}^{2}(\Omega)}\left(C_{d}\right.$ given by Lemma 5.3$)$, Inequalities (4.10) and (4.9) (and Poincaré Inequality) give the existence of $\bar{C}_{\epsilon}$ only depending on $\epsilon, f, \eta$ and $\Omega$, such that

$$
\left\|p_{l}-m\left(p_{l}\right)\right\|_{L^{2}(\Omega)} \leq \epsilon\|p\|_{L^{2}(\Omega)}+\bar{C}_{\epsilon} .
$$

As in Step 1, we now use $\int_{\Omega} \rho_{m} d x=M$ (and the fonction $\bar{\eta}$ ). Taking $\epsilon<1$ gives, by Lemma 5.4 , the existence of $C_{2}$, only depending on the $\Omega, f, \eta$ and $M$, such that

$$
\left\|p_{l}\right\|_{\mathrm{L}^{2}(\Omega)} \leq C_{2} \text {. }
$$

As in Remark 4.1, this estimate holds also if $\eta$ is nondecreasing (instead of increasing).

With (4.11), turning back to (4.9) and (4.8), there exists $C_{3}$, only depending on $\Omega$, $f, \eta$ and $M$, such that

$$
\left\|u_{l}\right\|_{H_{0}^{1}(\Omega)^{N}} \leq C_{3} \text { and }\left\|\rho_{l}\right\|_{L^{2}(\Omega)^{N}} \leq C_{3} .
$$

Since $n$ is fixed, we also obtain an $H^{1}(\Omega)$ estimate on $\rho_{l}$. Actually, Theorem 2.1 gives the existence of $C_{4}$ only depending on the $\Omega, f, \eta, M, \varphi$ and $n$ such that

$$
\left\|\rho_{l}\right\|_{H^{1}(\Omega)} \leq C_{4} \text {. }
$$

Thanks to the estimates obtained in this Step 2, it is possible to assume (up to a subsequence) that, as $l \rightarrow+\infty$ :

$$
\begin{aligned}
& \text { - } u_{l} \rightarrow u \text { in } L^{2}(\Omega)^{N} \text { and weakly in } H_{0}^{1}(\Omega)^{N}, \\
& \text { - } \rho_{l} \rightarrow \rho \text { a.e, in } L^{2}(\Omega) \text { and weakly in } H^{1}(\Omega) \text {, } \\
& \text { - } p_{l} \rightarrow p \text { weakly in } L^{2}(\Omega) \text {. }
\end{aligned}
$$

Then, passing to the limit (as $l \rightarrow+\infty$ ) in the equations satisfied by $\left(u_{l}, \rho_{l}, p_{l}\right)$ gives that $(u, p, \rho)$ is solution of (4.6). In particular, as in Step 1, since $\eta\left(\rho_{l}\right) \rightarrow \eta(\rho)$ a.e and $\left(\eta\left(\rho_{l}\right)\right)_{m \in \mathbb{N}}$ is bounded in $L^{2}(\Omega)$, one has $\eta\left(\rho_{l}\right) \rightarrow \eta(\rho)$ in $L^{q}(\Omega)$ for all $q<2$. Since $\eta\left(\rho_{l}\right)=p_{l} \rightarrow p$ weakly in $L^{2}(\Omega)$, we then conclude that $p=\eta(\rho)$. Here also, we recall that the fact that $\rho>0$ a.e. in $\Omega$ is given by Theorem 2.1. This concludes the proof of Step 2.

Step 3, $n \rightarrow+\infty$ 
Step 2 gives, for all $n \in \mathbb{N}^{*}$ the existence of $\left(u_{n}, \rho_{n}, p_{n}\right)$ such that

$$
\begin{aligned}
& u_{n} \in H_{0}^{1}(\Omega)^{N}, \rho_{n} \in H^{1}(\Omega), p_{n} \in L^{2}(\Omega), \\
& \int_{\Omega} \nabla u_{n}: \nabla v d x-\int_{\Omega} p_{n} \operatorname{div}(v) d x=\int_{\Omega} f\left(x, \rho_{n}\right) \cdot v d x, \forall v \in \mathrm{H}_{0}^{1}(\Omega)^{N}, \\
& \int_{\Omega} \varphi\left(\rho_{n}\right) u_{n} \cdot \nabla \psi d x-\frac{1}{n} \int_{\Omega} \nabla \rho_{n}(x) \cdot \nabla \psi(x) d x=0 \text { for all } \psi \in H^{1}(\Omega), \\
& \rho_{n}>0 \text { a.e. in } \Omega, \int_{\Omega} \rho_{n} d x=M, p_{n}=\eta\left(\rho_{n}\right) \text { a.e. in } \Omega .
\end{aligned}
$$

In order to obtain a solution of (1.5), we have to pass to the limit in these equations as $n \rightarrow+\infty$.

As in Step 2, we obtain an $H_{0}^{1}(\Omega)$ estimate on $u_{n}$ and an $L^{2}(\Omega)$ estimate on $\rho_{n}$ and $p_{n}$ (the proof is the same as in Step 2, replacing $f_{l}$ by $f$ ). Namely, there exists $C_{1}$ only depending on $\Omega, f, \eta$ and $M$, such that

$$
\left\|u_{n}\right\|_{H_{0}^{1}(\Omega)^{N}},\left\|\rho_{n}\right\|_{L^{2}(\Omega)},\left\|p_{n}\right\|_{L^{2}(\Omega)} \leq C_{1} .
$$

Thanks to these estimates, it is possible to assume (up to a subsequence) that, as $n \rightarrow+\infty$,

$$
\begin{aligned}
& \text { - } u_{n} \rightarrow u \text { in } L^{2}(\Omega)^{N} \text { and weakly in } H_{0}^{1}(\Omega)^{N}, \\
& \text { - } \rho_{n} \rightarrow \rho \text { weakly in } L^{2}(\Omega), \\
& \text { - } p_{n} \rightarrow p \text { weakly in } L^{2}(\Omega) \text {. }
\end{aligned}
$$

But, we do not have an $H^{1}(\Omega)$ estimate on $\rho_{n}$ (this estimate in Step 2 was depending on $n)$. So, we need some additional tricks to prove the convergence of $f\left(\cdot, \rho_{n}\right), \varphi\left(\rho_{n}\right)$ and $\eta\left(\rho_{n}\right)$ to $f(\cdot, \rho), \varphi(\rho)$ and $\eta(\rho)$.

We first remark that the weak convergence of $\rho_{n}$ in $L^{2}(\Omega)$ gives $\rho \geq 0$ a.e. and $\int_{\Omega} \rho d x=M$.

We now try to pass to the limit in (4.14b) and (4.14c). For $n \in \mathbb{N}^{*}$, we set $h_{n}=f\left(\cdot, \rho_{n}\right)$ and $q_{n}=\varphi\left(\rho_{n}\right)$. Thanks to Hypothesis (1.2) on $f$ and Hypothesis (1.4b) on $\varphi$, the sequences $\left(h_{n}\right)_{n \in \mathbb{N}^{*}}$ and $\left(q_{n}\right)_{n \in \mathbb{N}^{*}}$ are bounded in $L^{2}(\Omega)^{N}$ and $L^{2}(\Omega)$. Then, it is possible to assume (up to a subsequence) that, as $n \rightarrow+\infty$,

$$
\begin{aligned}
& \text { - } h_{n} \rightarrow h \text { weakly in } L^{2}(\Omega)^{N} \text {, } \\
& \text { - } q_{n} \rightarrow q \text { weakly in } L^{2}(\Omega) .
\end{aligned}
$$

Thanks to the convergence of $u_{n}, p_{n}$ and $h_{n}$, it is quite easy to pass to the limit, as $n \rightarrow \infty$, in (4.14b). We obtain

$$
\int_{\Omega} \nabla u: \nabla v d x-\int_{\Omega} p \operatorname{div}(v) d x=\int_{\Omega} h \cdot v d x, \forall v \in \mathrm{H}_{0}^{1}(\Omega)^{N} .
$$

We prove now that

$$
\int_{\Omega} q u \cdot \nabla \psi d x=0 \text { for all } \psi \in W^{1, \infty}(\Omega),
$$

Let $\epsilon>0$ and $n \in \mathbb{N}^{*}$. We take $\psi=\ln \left(\rho_{n}+\epsilon\right)$ in (4.14c) (this is possible since $\rho_{n}>0$ a.e. and then $\left.\psi \in H^{1}(\Omega)\right)$. We obtain

$$
\frac{1}{n} \int_{\Omega} \frac{\left|\nabla \rho_{n}(x)\right|^{2}}{\rho_{n}(x)+\epsilon} d x=\frac{1}{n} \int_{\Omega} \nabla \rho_{n}(x) \cdot \nabla \psi(x) d x=\int_{\Omega} \varphi\left(\rho_{n}(x)\right) u(x) \cdot \frac{\nabla \rho_{n}(x)}{\rho_{n}(x)+\epsilon} .
$$


We define $\phi$ by $\phi(s)=\int_{0}^{s} \frac{\varphi(\xi)}{\xi+\epsilon} d \xi$ for $s>0$. Thanks to (1.4b), one has $|\phi(s)| \leq L s$ (and $|\varphi(s)| \leq L s$ ) for all $s>0$. Since $\rho_{n} \in H^{1}(\Omega)$ and $\rho_{n}>0$ a.e., one has $\phi\left(\rho_{n}\right) \in H^{1}(\Omega)$ and the previous equality gives

$$
\frac{1}{n} \int_{\Omega} \frac{\left|\nabla \rho_{n}(x)\right|^{2}}{\rho_{n}(x)+\epsilon} d x=\int_{\Omega} u(x) \cdot \nabla \phi\left(\rho_{n}(x)\right) d x=\int_{\Omega} \phi\left(\rho_{n}(x)\right) \operatorname{div}(u(x)) d x .
$$

Then, thanks to (4.15), there exists $C_{2}$ only depending on $\Omega, f, \eta$ and $M$, such that

$$
\frac{1}{n} \int_{\Omega} \frac{\left|\nabla \rho_{n}(x)\right|^{2}}{\rho_{n}(x)+\epsilon} d x \leq C_{2}
$$

When $\epsilon \rightarrow 0$, this inequality gives, with the theorem of Monotone Convergence, for all $n \in \mathbb{N}^{*}$,

$$
\frac{1}{n} \int_{\Omega} \frac{\left|\nabla \rho_{n}(x)\right|^{2}}{\rho_{n}(x)} d x \leq C_{2}
$$

Inequality (4.18) allows us to prove (4.17). Indeed, let $\psi \in W^{1, \infty}(\Omega)$. Thanks to the convergence of $u_{n}$ to $u$ in $L^{2}(\Omega)$ and to the weak convergence of $\varphi\left(\rho_{n}\right)$ to $q$ in $L^{2}(\Omega)$, one has, as $n \rightarrow \infty$,

$$
\int_{\Omega} \varphi\left(\rho_{n}\right) u_{n} \cdot \nabla \psi d x \rightarrow \int_{\Omega} q u \cdot \nabla \psi d x
$$

and Inequality (4.18) gives

$$
\left|\frac{1}{n} \int_{\Omega} \nabla \rho_{n}(x) \cdot \nabla \psi(x) d x\right| \leq \frac{1}{\sqrt{n}} \sqrt{C_{2} M}\||\nabla \psi|\|_{L^{\infty}(\Omega)} \rightarrow 0 .
$$

Then, passing to the limit in (4.14c) (with $\psi \in W^{1, \infty}(\Omega)$ ) leads to (4.17).

The main difficulty now is that we do not know if $h=f(\cdot, \rho)$ and $q=\varphi(\rho)$ (except in the very interesting case where $f(x, \rho)=\bar{f}(x)+g(x) \rho(x)$, with $\bar{f} \in L^{2}(\Omega)$ and $\left.g \in L^{\infty}(\Omega)\right)$ and we do not know if $p=\eta(\rho)$ (at least, up to a subsequence). In order to prove these results, we will first show that

$$
\liminf _{n \rightarrow \infty} \int_{\Omega} p_{n} q_{n} d x \leq \int_{\Omega} p q d x
$$

Since the sequence $\left(q_{n}\right)_{n \in \mathbb{N}}$ is bounded in $\mathrm{L}^{2}(\Omega)$, Lemma 5.5 gives the existence of a bounded sequence $\left(v_{n}\right)_{n \in \mathbb{N}}$ in $\mathrm{H}^{1}(\Omega)^{N}$ such that $\operatorname{div}\left(v_{n}\right)=q_{n}$ and $\operatorname{curl}\left(v_{n}\right)=0$. It is possible to assume (up to a subsequence) that $v_{n} \rightarrow v$ in $\mathrm{L}^{2}(\Omega)^{N}$ and weakly in $\mathrm{H}^{1}(\Omega)^{N}$. Passing to the limit as $n \rightarrow \infty$ gives $\operatorname{div}(v)=q$ and $\operatorname{curl}(v)=0$.

Let $\psi \in C_{c}^{\infty}(\Omega)$ (so that $\left.v_{n} \psi \in \mathrm{H}_{0}^{1}(\Omega)^{N}\right)$. Taking $v=v_{n} \psi$ in (4.14b) leads to

$$
\int_{\Omega} \nabla u_{n}: \nabla\left(v_{n} \psi\right) d x-\int_{\Omega} p_{n} \operatorname{div}\left(v_{n} \psi\right) d x=\int_{\Omega} h_{n} \cdot\left(v_{n} \psi\right) d x .
$$

Since $u_{n}, v_{n} \psi \in H_{0}^{1}(\Omega)^{N}$ one has

$$
\int_{\Omega} \boldsymbol{\nabla} u_{n}: \nabla v_{n} \psi d x=\int_{\Omega} \operatorname{curl}\left(u_{n}\right) \cdot \operatorname{curl}\left(v_{n} \psi\right) d x+\int_{\Omega} \operatorname{div}\left(u_{n}\right) \operatorname{div}\left(v_{n} \psi\right) d x .
$$

Thus,

$$
\begin{aligned}
\int_{\Omega} \operatorname{div}\left(u_{n}\right) \operatorname{div}\left(v_{n} \psi\right) d x+\int_{\Omega} \operatorname{curl}\left(u_{n}\right) & \cdot \operatorname{curl}\left(v_{n} \psi\right) d x \\
& -\int_{\Omega} p_{n} \operatorname{div}\left(v_{n} \psi\right) d x=\int_{\Omega} h_{n} \cdot\left(v_{n} \psi\right) d x .
\end{aligned}
$$


The choice of $v_{n}$ gives $\operatorname{div}\left(v_{n} \psi\right)=q_{n} \psi+v_{n} \cdot \nabla \psi$ and $\operatorname{curl}\left(v_{n} \psi\right)=L(\psi) v_{n}$, where $L(\psi)$ is a matrix with entries involving the first order derivatives of $\psi$. Then, the previous equality yields

$$
\begin{aligned}
\int_{\Omega}\left(\operatorname{div}\left(u_{n}\right)-\right. & \left.p_{n}\right) q_{n} \psi d x+\int_{\Omega} \operatorname{div}\left(u_{n}\right) v_{n} \cdot \nabla \psi d x \\
& +\int \operatorname{curl}\left(u_{n}\right) \cdot L(\psi) v_{n} d x-\int_{\Omega} p_{n} v_{n} \cdot \nabla \psi d x=\int_{\Omega} h_{n} \cdot\left(v_{n} \psi\right) d x .
\end{aligned}
$$

Thanks to the weak convergence of $u_{n}$ in $\mathrm{H}_{0}^{1}(\Omega)^{N}$ to $u$, the weak convergence of $p_{n}$ in $\mathrm{L}^{2}(\Omega)$ to $p$, the weak convergence of $h_{n}$ in $\mathrm{L}^{2}(\Omega)$ to $h$ and the convergence of $v_{n}$ in $\mathrm{L}^{2}(\Omega)^{N}$ to $v$, we obtain:

$$
\begin{aligned}
\lim _{n \rightarrow \infty} \int_{\Omega}\left(\operatorname{div}\left(u_{n}\right)-p_{n}\right) q_{n} \psi d x & =\int_{\Omega} h \cdot(v \psi) d x-\int_{\Omega} \operatorname{div}(u) v \cdot \nabla \psi d x \\
& -\int_{\Omega} \operatorname{curl}(u) \cdot L(\psi) v d x+\int_{\Omega} p v \cdot \nabla \psi d x .
\end{aligned}
$$

But, thanks to $(4.16),(u, p)$ satisfies:

$$
\int_{\Omega} \boldsymbol{\nabla} u: \nabla(v \psi) d x-\int_{\Omega} p \operatorname{div}(v \psi) d x=\int_{\Omega} h \cdot(v \psi) d x
$$

or equivalently:

$$
\int_{\Omega} \operatorname{div}(u) \operatorname{div}(v \psi) d x+\int_{\Omega} \operatorname{curl}(u) \cdot \operatorname{curl}(v \psi) d x-\int_{\Omega} p \operatorname{div}(v \psi) d x=\int_{\Omega} h \cdot(v \psi) d x,
$$

which gives (using $\operatorname{div}(v)=q$ and $\operatorname{curl}(v)=0$ )

$$
\begin{aligned}
\int_{\Omega}(\operatorname{div}(u)-p) q \psi d x+\int_{\Omega} \operatorname{div}(u) v \cdot \nabla \psi d x & +\int_{\Omega} \operatorname{curl}(u) \cdot L(\psi) v d x \\
& -\int_{\Omega} p v \cdot \nabla \psi d x=\int_{\Omega} h \cdot(v \psi) d x .
\end{aligned}
$$

Then, with (4.19), we obtain:

$$
\lim _{n \rightarrow \infty} \int_{\Omega}\left(p_{n}-\operatorname{div}\left(u_{n}\right)\right) q_{n} \psi d x=\int_{\Omega}(p-\operatorname{div}(u)) q \psi d x .
$$

In (4.20), the function $\psi$ is an arbitrary element of $\mathrm{C}_{c}^{\infty}(\Omega)$. We are going to prove now that it is possible to take $\psi=1$ in this relation.

Let $a>0$. Thanks to (1.4b) and (1.3b), there exists $b>0$ such, a.e. on $\Omega$,

$$
a q_{n}=a \varphi\left(\rho_{n}\right) \leq \eta\left(\rho_{n}\right)+b=p_{n}+b,
$$

so that

$$
q_{n}^{2} \leq \frac{2 p_{n}^{2}}{a^{2}}+\frac{2 b^{2}}{a^{2}} .
$$

If $C$ is a bound for the $L^{2}$-norm of $p_{n}$ (we already have such a bound), one obtains for any borelian subset $A$ of $\Omega$,

$$
\int_{A} q_{n}^{2} d x \leq \frac{2 C^{2}}{a^{2}}+\frac{2 b^{2}}{a^{2}}|A| .
$$

Let $\varepsilon>0$, we then take $a^{2}=2 C^{2} / \varepsilon$ which yields:

$$
\int_{A} \rho_{n}^{2} d x \leq \varepsilon+\frac{2 b^{2}}{a^{2}}|A| .
$$


and then, with $\delta=\frac{\varepsilon a^{2}}{2 b^{2}}$,

$$
|A| \leq \delta \Rightarrow \int_{A} q_{n}^{2} d x \leq 2 \varepsilon
$$

This proves the equi-integrability of the sequence $\left(q_{n}^{2}\right)_{n \in \mathbb{N}}$. Since the sequence $\left(\left(\operatorname{div}_{h_{n}} \boldsymbol{u}_{n}-p_{n}\right)\right)_{n \in \mathbb{N}}$ is bounded in $\mathrm{L}^{2}(\Omega)$, we then easily conclude (with the CauchySchwarz inequality) that the sequence $\left(\left(\operatorname{div}_{h_{n}} \boldsymbol{u}_{n}-p_{n}\right) q_{n}\right)_{n \in \mathbb{N}}$ is equi-integrable. Thus Lemma 5.7 yields the conclusion, namely (4.20) is true for $\psi=1$ a.e. on $\Omega$ :

$$
\lim _{n \rightarrow \infty} \int_{\Omega}\left(\operatorname{div}\left(u_{n}\right)-p_{n}\right) q_{n} d x=\int_{\Omega}(\operatorname{div}(u)-p) q d x .
$$

We now use the fact that $\varphi$ is continuous and nondecreasing. It gives, by Lemma 5.3 with $\phi=\varphi$, that

$$
\int_{\Omega} q_{n} \operatorname{div}\left(u_{n}\right) d x=\int_{\Omega} \varphi\left(\rho_{n}\right) \operatorname{div}\left(u_{n}\right) d x \leq 0
$$

and then

$$
\int_{\Omega} p_{n} q_{n} d x=\int_{\Omega}\left(p_{n}-\operatorname{div}\left(u_{n}\right)\right) q_{n} d x+\int_{\Omega} \operatorname{div}\left(u_{n}\right) q_{n} d x \leq \int_{\Omega}\left(p_{n}-\operatorname{div}\left(u_{n}\right)\right) q_{n} d x .
$$

With (4.21) it yields

$$
\limsup _{n \rightarrow \infty} \int_{\Omega} p_{n} q_{n} d x \leq \int_{\Omega}(p-\operatorname{div}(u)) q d x .
$$

We now recall that $(q, u)$ satisfy (4.17). One has $u \in H_{0}^{1}(\Omega), q \in L^{2}(\Omega)$ and $q \geq 0$ a.e. (since $\varphi$ is nondecreasing and then $q_{n}=\varphi\left(\rho_{n}\right) \geq 0$ a.e.). Then, Lemma 5.8 gives $\int_{\Omega} q \operatorname{div}(u) d x=0$ which gives

$$
\limsup _{n \rightarrow \infty} \int_{\Omega} p_{n} q_{n} d x \leq \int_{\Omega} p q d x
$$

and, up to a subsequence, we can assume

$$
\lim _{n \rightarrow \infty} \int_{\Omega} p_{n} q_{n} d x \leq \int_{\Omega} p q d x
$$

We now use the fact that $\varphi$ and $\eta$ are increasing. The function $\eta$ is a one-to-one function from $\mathbb{R}$ onto $\mathbb{R}$. We denote by $\bar{\eta}$ the reciprocal function of $\eta$ (that is $\bar{\eta}(\eta(s))=\eta(\bar{\eta}(s))=s$ for all $\left.s \in \mathbb{R}_{+}\right)$. Thanks to (1.3b), there exists $b \in \mathbb{R}$ such that $0 \leq \bar{\eta}(s) \leq s+b$ for all $s \in \mathbb{R}_{+}$. Then $\bar{\eta}(p) \in L^{2}(\Omega)$ (since $p \in L^{2}(\Omega)$ ). We set $\bar{\rho}=\bar{\eta}(p)$ (so that $\eta(\bar{\rho})=p)$ and $G_{n}=\left(\varphi\left(\rho_{n}\right)-\varphi(\bar{\rho})\right)\left(\eta\left(\rho_{n}\right)-\eta(\bar{\rho})\right)$ so that $G_{n} \in L^{1}(\Omega), G_{n} \geq 0$ a.e. and

$$
0 \leq \int_{\Omega} G_{n} d x=\int_{\Omega} q_{n} p_{n} d x-\int_{\Omega} q_{n} p d x-\int_{\Omega} \varphi(\bar{\rho}) p_{n} d x+\int_{\Omega} \varphi(\bar{\rho}) p d x .
$$

Thanks to (4.22) and to the $L^{2}(\Omega)$ weak convergences of $q_{n}$ and $p_{n}$ to $p$ and $q$, passing to the limit, as $n \rightarrow \infty$, in the previous inequality leads to

$$
0 \leq \lim _{n \rightarrow \infty} \int_{\Omega} G_{n} d x=\int_{\Omega}(q-\varphi(\bar{\rho}))(p-p) d x=0 .
$$

This gives $G_{n} \rightarrow 0$ in $L^{1}(\Omega)$ and then, up to a subsequence,

$$
G_{n}=\left(\varphi\left(\rho_{n}\right)-\varphi(\bar{\rho})\right)\left(\eta\left(\rho_{n}\right)-\eta(\bar{\rho})\right) \rightarrow 0 \text { a.e. in } \Omega .
$$


Thanks to the fact that $\varphi$ and $\eta$ are increasing, a classical argument by contradiction gives, from (4.23), that $\rho_{n} \rightarrow \bar{\rho}$ a.e. in $\Omega$ as $n \rightarrow \infty$ (see, for instance [5]). Since the sequence $\left(\rho_{n}\right)_{n \in \mathbb{N}}$ is bounded in $L^{2}(\Omega)$, we then conclude that $\rho_{n} \rightarrow \bar{\rho}$ in $L^{q}(\Omega)$ for all $q<2$. But, we already know that $\rho_{n} \rightarrow \rho$ weakly in $L^{2}(\Omega)$ (and therefore weakly in $L^{q}(\Omega)$ for $\left.q \leq 2\right)$. Then, the uniqueness of the weak limit gives $\bar{\rho}=\rho$ a.e.. Therefore, we also obtain that $q=\varphi(\rho)$ a.e., $h=f(\cdot, \rho)$ a.e. and $p=\eta(\rho)$ a.e. and this proves that $(u, \rho, p)$ is solution of (1.5). This concludes the proof of Theorem 1.3.

The following theorem (Theorem 4.2) is devoted to the important case where $f(x, \rho)=\bar{f}(x)+g(x) \rho(x)$ and $\varphi(\rho)=\rho)$. In this case, the hypothesis " $\eta$ is increasing" can be replaced by the weaker hypothesis " $\eta$ is nondecreasing".

Theorem 4.2. Let $M>0$ and $\Omega$ be a connected bounded open set of $\mathbb{R}^{N}(N \geq 1)$ with a Lipschitz continuous boundary. Assume that $f(x, \rho)=\bar{f}(x)+g(x) \rho(x)$, with $\bar{f} \in L^{2}(\Omega)^{N}$ and $g \in L^{\infty}(\Omega)^{N}$. Assume that $\eta$ satisfy (1.3) but with $\eta$ nondecreasing instead of $\eta$ increasing. Then (1.5) has at least one solution.

\section{Proof}

In order to prove this theorem, there are some minor changes to do in the proof of Theorem 1.3.

In Step 1 of the proof of Theorem 1.3, we have to change the definition of $\bar{\eta}$ in order to have the estimate on $p_{m}$. We take, for $\bar{\eta}$, the reciprocal function of the function $s \mapsto \eta(s)+s$ (which is one-to-one from $\mathbb{R}_{+}$onto $\mathbb{R}_{+}$), as it is explained in remark 4.1 .

In Step 3, since $\rho_{n} \rightarrow \rho$ weakly in $L^{2}(\Omega)$, as $n \rightarrow \infty$, the hypotheses on $\varphi$ and $f$ give $q=\rho$ and $h=f(\cdot, \rho)$. Then it remains only to prove that $p=\eta(\rho)$.

Thanks to fact that $\eta$ is nondecreasing, we now use the Minty-trick. In order to have $\eta$ defined on the whole $\mathbb{R}$, we set $\eta(s)=s$ for $s<0$. Let $\bar{\rho} \in L^{2}(\Omega)$ such that $\eta(\bar{\rho}) \in L^{2}(\Omega)$. Since $\rho_{n}, \bar{\rho}, \eta\left(\rho_{n}\right), \eta(\bar{\rho}) \in L^{2}(\Omega)$ one has, for all $n \in \mathbb{N}^{*}$,

$$
0 \leq \int_{\Omega}\left(\rho_{n}-\bar{\rho}\right)\left(\eta\left(\rho_{n}\right)-\eta(\bar{\rho})\right) d x=\int_{\Omega}\left(\rho_{n}-\bar{\rho}\right)\left(p_{n}-\eta(\bar{\rho})\right) d x .
$$

Thanks to (4.22) and to the $L^{2}(\Omega)$ weak convergences of $\rho_{n}$ and $p_{n}$ to $\rho$ and $p$, passing to the limit, as $n \rightarrow \infty$, in the previous inequality leads to

$$
0 \leq \int_{\Omega}(\rho-\bar{\rho})(p-\eta(\bar{\rho})) d x
$$

which gives also

$$
0 \leq \int_{\Omega}(\rho-\bar{\rho})(p+\rho-\eta(\bar{\rho})-\bar{\rho}) d x
$$

Let $\bar{\eta}$ be the reciprocal function of the function $s \mapsto \eta(s)+s$ (which is a one-to-one function from $\mathbb{R}$ onto $\mathbb{R}$ ). Let $\bar{p} \in L^{2}(\Omega)$. Since $0 \leq \bar{\eta}(s) \leq s$ for all $s \in \mathbb{R}_{+}$, one has $\bar{\eta}(\bar{p}) \in L^{2}(\Omega)$ and we can take $\bar{\rho}=\bar{\eta}(\bar{p})$ in $(4.25)$, it yields

$$
0 \leq \int_{\Omega}(\rho-\bar{\eta}(\bar{p}))(p+\rho-\bar{p}) d x .
$$


Let $\psi \in C_{c}^{\infty}(\Omega)$ and $\epsilon>0$. Taking $\bar{p}=p+\rho+\epsilon \psi$ in (4.26) and letting $\epsilon \rightarrow 0$ leads to, with the Dominated Convergence Theorem,

$$
0 \leq-\int_{\Omega}(\rho-\bar{\eta}(p+\rho)) \psi d x
$$

Since $\psi$ is arbitrary in $C_{c}^{\infty}(\Omega)$, we then conclude that $\rho=\bar{\eta}(p+\rho)$ a.e. and, finally, $p=\eta(\rho)$ a.e.. This concludes Theorem 4.2.

\section{Some Lemmas}

Lemma 5.1. Let $\Omega$ be a connected bounded open set of $\mathbb{R}^{N}(N \geq 1)$ with a Lipschitz continuous boundary. Let $\omega \subset \Omega$ be a measurable set with positive Lebesgue measure. We define the set $W_{\omega}$ by:

$$
W_{\omega}=\left\{u \in W^{1,1}(\Omega) \text { such that } u=0 \text { a.e. in } \omega\right\} .
$$

Then there exist $C$ only depending on $\Omega$ and $\omega$ such that

$$
\|u\|_{L^{p}(\Omega)} \leq C\||\nabla u|\|_{L^{1}(\Omega)} \text { for all } u \in W_{\omega} \text { and for all } 1 \leq p \leq \frac{N}{N-1} .
$$

Proof

Since $\Omega$ is bounded, we only have to prove (5.1) for $p=1^{\star}=N /(N-1)$. With the Sobolev Embedding Theorem, we already know that there exist $C_{1}$ only depending on $\Omega$ such that $\|u\|_{L^{1^{\star}}(\Omega)} \leq C_{1}\|u\|_{W^{1,1}(\Omega)}$ for all $u \in W^{1,1}(\Omega)$. Then we only have to show that on $W_{\omega}$ the $W^{1,1}$-norm of $u$ is equivalent to the $L^{1}$-norm of the gradient of $u$, that is that there exists $C_{2}$ only depending on $\Omega$ and $\omega$ such that

$$
\|u\|_{L^{1}(\Omega)} \leq C_{2}\||\nabla u|\|_{L^{1}(\Omega)} \text { for all } u \in W_{\omega} .
$$

In order to prove the existence of $C_{2}$ such that (5.2) holds, we argue by contradiction. We assume the existence of a sequence $\left(u_{n}\right)_{n \in \mathbb{N}^{\star}}$ in $W_{\omega}$ such that

$$
\left\|u_{n}\right\|_{L^{1}(\Omega)} \geq n\left\|\left|\nabla u_{n}\right|\right\|_{L^{1}(\Omega)} \text { for all } n \in \mathbb{N}^{\star} .
$$

Replacing $u_{n}$ by $u_{n} /\left\|u_{n}\right\|_{L^{1}(\Omega)}$, we can assume that $\left\|u_{n}\right\|_{L^{1}(\Omega)}=1$. Then, $\left(u_{n}\right)_{n \in \mathbb{N}^{*}}$ is bounded in $W^{1,1}(\Omega)$ and it is relatively compact in $L^{1}(\Omega)$ (by Rellich' Theorem). Therefore, we can assume (up to a subsequence) that $u_{n} \rightarrow u$ in $L^{1}(\Omega)$ and a.e..

Furthermore, since

$$
\left\|\left|\nabla u_{n}\right|\right\|_{L^{1}(\Omega)} \leq \frac{1}{n}
$$

one has $\boldsymbol{\nabla} u=0$ a.e. in $\Omega$ and, since $\Omega$ is connected, $u$ is a constant function. Then, the fact that $u_{n}=0$ a.e. in $\omega$ gives that $u=0$ a.e. in $\omega$. Therefore $u=0$ a.e. in $\Omega$. But, this is impossible since $u_{n} \rightarrow u$ in $L^{1}(\Omega)$ and $\left\|u_{n}\right\|_{L^{1}(\Omega)}=1$. This concludes the proof of Lemma 5.1 .

N.B. It is also possible to prove Lemma 5.1 using the "mean-value" Sobolev Inequality (or also using the Poincaré-Wirtinger Inequality). Actually, there exists $C_{s}$ only depending on $\Omega$ such that for all $u \in W^{1,1}(\Omega)$ one has, with $m \lambda_{N}(\Omega)=\int_{\Omega} u(x) d x$,

$$
\|u-m\|_{L^{1^{\star}}(\Omega)} \leq C_{s}\||\nabla u|\|_{L^{1}(\Omega)} .
$$

Then, for $u \in W_{\omega}$, since gives

$$
\int_{\Omega \backslash \omega}|u-m|^{1^{\star}} d x+|m|^{1^{\star}} \lambda_{N}(\omega) \leq C_{s}^{1^{\star}}\||\nabla u|\|_{L^{1}(\Omega)}^{1^{\star}} .
$$


Then, we have $|m| \leq \frac{C_{s}}{\lambda_{N}(\omega)^{1 / 1^{\star}}}\||\nabla u|\|_{L^{1}(\Omega)}$, and we conclude by using

$$
\|u\|_{L^{1^{\star}}(\Omega)} \leq\|u-m\|_{L^{1^{\star}}(\Omega)}+|m| \lambda_{N}(\Omega)^{1 / 1^{\star}} \leq C_{s}\left(1+\left(\frac{\lambda_{N}(\Omega)}{\lambda_{N}(\omega)}\right)^{1 / 1^{\star}}\right)\||\nabla u|\|_{L^{1}(\Omega)} .
$$

Lemma 5.2. Let $\Omega$ be a connected bounded open set of $\mathbb{R}^{N}(N=2$ or 3$)$ with a Lipschitz continuous boundary. Let $\varphi$ be a function from $\mathbb{R}$ to $\mathbb{R}$ satisfying (1.4b) and such that $\varphi(s)>0$ if $s>0$. Let $n \in \mathbb{N}^{\star}, M>0, u \in \mathrm{H}_{0}^{1}(\Omega)^{N}$ and $\rho \in H^{1}(\Omega)$ a solution of the following problem:

$$
\begin{aligned}
& \int_{\Omega} \varphi(\rho(x)) u(x) \cdot \nabla \psi(x) d x-\frac{1}{n} \int_{\Omega} \nabla \rho(x) \cdot \nabla \psi(x) d x=0 \forall \psi \in H^{1}(\Omega), \\
& \int_{\Omega} \rho(x) d x=M .
\end{aligned}
$$

Then, $\rho>0$ and for $\Phi \in C^{0}\left(\mathbb{R}^{+}, \mathbb{R}\right)$ nondecreasing such that $\Phi(0)=0$ and $\Phi(\rho) \in$ $L^{2}(\Omega)$,

$$
\int_{\Omega} \Phi(\rho) \operatorname{div}(u) d x \leq 0
$$

\section{Proof}

The positivity of the solution $\rho$ of (5.3) results from theorem 2.1 .

We now give the proof of (5.4) which is composed of four steps.

Step 1 Let $\alpha, \beta, \gamma \in \mathbb{R}_{+}^{*}, \beta<\gamma$. We suppose in this case that $\Phi$ satisfies the following properties:

$$
\left\{\begin{array}{l}
\Phi \in C^{1}(\mathbb{R}), \text { nondecreasing } \\
\Phi=0 \text { on }]-\infty, \beta] \\
\Phi=\alpha \text { on }] \gamma,+\infty[
\end{array}\right.
$$

Let $\Psi$ defined by $\Psi(s)=\int_{0}^{s} \frac{\Phi^{\prime}(t)}{\varphi(t)} \mathrm{d} t$.

Taking $\psi=\Psi \circ \rho \in H^{1}(\Omega)$ in equation (5.3), that is

$$
\int_{\Omega} \varphi(\rho) u \cdot \nabla \psi d x-\frac{1}{n} \int_{\Omega} \nabla \rho \cdot \nabla \psi d x=0
$$

we obtain

$$
\int_{\Omega} u \cdot \Phi^{\prime}(\rho) \nabla \rho d x-\frac{1}{n} \int_{\Omega}|\nabla \rho|^{2} \frac{\Phi^{\prime}(\rho)}{\varphi(\rho)} d x=0
$$

Using the fact that the function $\Phi$ is nondecreasing and $\rho>0$, we get

$$
\int_{\Omega} u \Phi^{\prime}(\rho) \nabla \rho d x \geq 0
$$

and then since $u \in H_{0}^{1}(\Omega)^{d}$,

$$
\int_{\Omega} \Phi(\rho) \operatorname{div}(u) d x \leq 0
$$


Step 2 In this step, we also have $\alpha, \beta, \gamma \in \mathbb{R}_{+}^{*}, \beta<a$ and we take $\Phi$ satisfying

$$
\left\{\begin{array}{l}
\Phi \in C^{0}(\mathbb{R}), \text { nondecreasing, } \\
\Phi=0 \text { on }]-\infty, \beta], \\
\Phi=\alpha \text { on }[\gamma,+\infty[.
\end{array}\right.
$$

Let $\left(\alpha_{m}\right)_{m \in \mathbb{N}^{\star}} \in C_{c}^{\infty}(\mathbb{R})$ be a sequence of mollifiers, that is $\alpha_{m}(s)=m \alpha(m s)$ (for $\left.s \in \mathbb{R}, m \in \mathbb{N}^{*}\right), \alpha \in C_{c}^{\infty}\left(\mathbb{R}, \mathbb{R}_{+}\right), \alpha(s)=0$ if $|s| \geq 1$ and $\int_{\mathbb{R}} \alpha d x=1$.

Let $\Phi_{m}=\Phi * \alpha_{m}$. We then get by Step 1 , if $1 / m<\beta$

and then using the fact that

$$
\int_{\Omega} \Phi_{m}(\rho) \operatorname{div}(u) d x \leq 0
$$

$$
\Phi_{m} \rightarrow \Phi \text { a.e in } \mathbb{R}
$$

and

$$
\left\|\Phi_{m}\right\|_{L^{\infty}} \leq\|\Phi\|_{L^{\infty}}\left\|\alpha_{m}\right\|_{L^{1}}=\|\Phi\|_{L^{\infty}}
$$

we thus get applying the Dominated Convergence Theorem, as $m \rightarrow+\infty$,

$$
\int_{\Omega} \Phi(\rho) \operatorname{div}(u) d x \leq 0
$$

Step 3 In this step we take $\alpha, \gamma \in \mathbb{R}_{+}^{\star}$ and $\Phi$ satisfying

$$
\left\{\begin{array}{l}
\Phi \in C^{0}(\mathbb{R}), \text { nondecreasing, } \\
\Phi=0 \text { on }]-\infty, 0] \\
\Phi=\alpha \text { on }[a,+\infty[.
\end{array}\right.
$$

Let $\beta>0$ and $\Phi_{\beta}$ defined by $\Phi_{\beta}(x)=\Phi(x-\beta)$ so that $\Phi_{\beta}=0$ on $\left.]-\infty, \beta\right]$. Then by Step 2 we get

$$
\int_{\Omega} \Phi_{\eta}(\rho) \operatorname{div}(u) d x \leq 0
$$

Finally, applying the Dominated Convergence Theorem, we get as $\beta \rightarrow 0$

$$
\int_{\Omega} \Phi(\rho) \operatorname{div}(u) d x \leq 0
$$

Step 4 In this step we take $\Phi$ satisfying

$$
\left\{\begin{array}{l}
\Phi \in C^{0}(\mathbb{R}), \text { nondecreasing, } \\
\Phi(0)=0 \\
\Phi(\rho) \in L^{2}(\Omega) .
\end{array}\right.
$$

Let $n \in \mathbb{N}^{*}$ we define $\Phi_{n}$ by $\Phi_{n}(s)=\min \left(n, \Phi\left(s^{+}\right)\right)$where $s^{+}=\max (0, s)$. By Step 3 we have

$$
\int_{\Omega} \Phi_{n}(\rho) \operatorname{div}(u) d x \leq 0 .
$$

Since $\rho>0$ and $\Phi(\rho) \in L^{2}(\Omega)$, applying the Dominated Convergence Theorem we pass to the limit as $n \rightarrow+\infty$ and we get

$$
\int_{\Omega} \Phi(\rho) \operatorname{div}(u) d x \leq 0
$$

The following lemma is well-known. A simple proof of this result is given in [2]. 
Lemma 5.3. Let $\Omega$ be a bounded open subset of $\mathbb{R}^{N}(N \geq 1)$ with a Lipschitz continuous boundary. Let $q \in \mathrm{L}^{2}(\Omega)$ such that $\int_{\Omega} q d x=0$. Then, there exists $w \in \mathrm{H}_{0}^{1}(\Omega)^{N}$ such that $\operatorname{div}(w)=q$ a.e. in $\Omega$ and $\|w\|_{H_{0}^{1}(\Omega)^{N}} \leq C_{d}\|q\|_{L^{2}(\Omega)}$ where $C_{d}$ only depends on $\Omega$.

Lemma 5.4. Let $\Omega$ be a bounded set of $\mathbb{R}^{N}(N \geq 1)$ and $p \in L^{2}(\Omega), p \geq 0$ a.e.. We assume that there exist $0 \leq a<1$ and $b \in \mathbb{R}$ such that

$$
\|p-m\|_{L^{2}(\Omega)}=a\|p\|_{L^{2}(\Omega)}+b,
$$

where $m$ is the mean value of $p$. Furthermore, we assume that there exist $A \in$ $\mathbb{R}$ and a continuous function $\theta$ from $\mathbb{R}^{+}$to $\mathbb{R}^{+}$such that $\int_{\Omega} \theta(p) d x \leq A$ and $\lim _{s \rightarrow+\infty} \theta(s)=+\infty$. Then, there exists $C$ only depending on $\Omega, a, b, A$ and $\theta$ such that

$$
\|p\|_{L^{2}(\Omega)} \leq C .
$$

The proof of Lemma 5.4 is in [7].

Lemma 5.5. Let $\Omega$ be a bounded open set of $\mathbb{R}^{N}$ and $q \in \mathrm{L}^{2}(\Omega)$. Then, there exists $v \in \mathrm{H}^{1}(\Omega)^{N}$ such that $\operatorname{div}(v)=q$ a.e. in $\Omega, \operatorname{curl}(v)=0$ a.e. in $\Omega$ and $\|v\|_{\mathrm{H}^{1}(\Omega)^{N}} \leq C\|q\|_{\mathrm{L}^{2}(\Omega)}$ where $C$ only depends on $\Omega$.

Proof

This lemma is very classical. For instance, it is possible to take $v=\boldsymbol{\nabla} w$ where $w \in H_{0}^{1}(\Omega)$ is the weak solution of $\Delta w=q$ in a ball $B$ containing $\Omega$ (see, for instance, [5]).

Definition 5.6. Let $\Omega$ be a subset of $\mathbb{R}^{N}$. A sequence $\left(F_{n}\right)_{n \in \mathbb{N}} \subset \mathrm{L}^{1}(\Omega)$ is said equi-integrable if

$$
\lim _{\lambda_{N}(A) \rightarrow 0} \int_{A}\left|F_{n}\right| d x=0 \text {, uniformly with respect to } n \in \mathbb{N},
$$

where $\lambda_{N}(A)$ denotes the $N$-dimensional Lebesgue measure of the Borelian subset $A \subset \Omega$.

Lemma 5.7. Let $\Omega$ be a bounded open subset of $\mathbb{R}^{N}$. Let $\left(F_{n}\right)_{n \in \mathbb{N}} \subset \mathrm{L}^{1}(\Omega)$ be an equi-integrable sequence, and $F$ be a function of $\mathrm{L}^{1}(\Omega)$. We assume that:

$$
\lim _{n \rightarrow \infty} \int_{\Omega} F_{n} \varphi d x=\int_{\Omega} F \varphi d x \text { for all } \varphi \in \mathrm{C}_{c}^{\infty}(\Omega)
$$

Then

$$
\lim _{n \rightarrow \infty} \int_{\Omega} F_{n} d x=\int_{\Omega} F d x
$$

Lemma 5.7 is well-known. A proof is given, for instance, in [5].

Lemma 5.8. Let $\Omega$ be a bounded open subset of $\mathbb{R}^{N}$. Let $q \in L^{2}(\Omega), q \geq 0$ a.e. in $\Omega$ and $u \in H_{0}^{1}(\Omega)^{N}$. Assume that $(q, u)$ satisfies:

$$
\int_{\Omega} q u \cdot \nabla \psi d x=0 \text { for all } \psi \in W^{1, \infty}(\Omega) .
$$

Then,

$$
\int_{\Omega} q \operatorname{div}(u) d x=0
$$


See [5] for a proof of Lemma 5.8.

\section{REFERENCES}

[1] H. Bijl and P. Wesseling. A unified method for computing incompressible and compressible flows in boundary-fitted coordinates. J. Comput. Phys., 141(2):153-173, 1998.

[2] J.H. Bramble. A proof of the inf-sup condition for the Stokes equations on Lipschitz domains. Mathematical Models and Methods in Applied Sciences, 13:361-371, 2003.

[3] J. Březina and A. Novotný. On weak solutions of steady Navier-Stokes equations for monatomic gas. Comment. Math. Univ. Carolin., 49(4):611-632, 2008.

[4] J. Droniou and J.-L. Vázquez. Noncoercive convection-diffusion elliptic problems with Neumann boundary conditions. Calc. Var. Partial Differential Equations, 34(4):413-434, 2009.

[5] R. Eymard, T. Gallouët, R. Herbin, and J. C. Latché. A convergent finite element-finite volume scheme for the compressible Stokes problem. II. The isentropic case. Math. Comp., 79(270):649-675, 2010.

[6] E. Feireisl. Dynamics of viscous compressible flows. volume 26 of Oxford Lecture Series in Mathematics and its Applications. Oxford University Press, 2004.

[7] A. Fettah and T. Gallouët. Numerical approximation of the general compressible Stokes problem. IMA J. Numer. Anal., 33(3):922-951, 2013.

[8] J. Frehse, M. Steinhauer, and W. Weigant. The Dirichlet problem for viscous compressible isothermal Navier-Stokes equations in two dimensions. Arch. Ration. Mech. Anal., 198(1):112,2010 .

[9] J. Frehse, M. Steinhauer, and W. Weigant. The Dirichlet problem for steady viscous compressible flow in three dimensions. J. Math. Pures Appl. (9), 97(2):85-97, 2012.

[10] T. Gallouët and R. Herbin. Mesure, intégration, probabilités. Ellipses, 2013.

[11] T. Gallouët, R. Herbin, and J.-C. Latché. A convergent finite element-finite volume scheme for the compressible Stokes problem. I. The isothermal case. Math. Comp., 78(267):1333-1352, 2009.

[12] F. Harlow and A. Amsden. A numerical fluid dynamics calculation method for all flow speeds. Journal of Computational Physics, 8:197-213, 1971.

[13] D. Jesslé and A. Novotný. Existence of renormalized weak solutions to the steady equations describing compressible fluids in barotropic regime. J. Math. Pures Appl. (9), 99(3):280-296, 2013.

[14] S. Jiang and C. Zhou. Existence of weak solutions to the three-dimensional steady compressible Navier-Stokes equations. Ann. Inst. H. Poincaré Anal. Non Linéaire, 28(4):485-498, 2011.

[15] J. Leray. Sur le mouvement d'un liquide visqueux emplissant l'espace. Acta Math., 63(1):193248,1934

[16] P.-L. Lions. Mathematical topics in fluid mechanics - volume 2 - compressible models. volume 10 of Oxford Lecture Series in Mathematics and its Applications. Oxford University Press, 1998.

[17] S. Novo and A. Novotný. On the existence of weak solutions to the steady compressible Navier-Stokes equations when the density is not square integrable. J. Math. Kyoto Univ., $42(3): 531-550,2002$.

[18] A. Novotný and I. Straškraba. Introduction to the mathematical theory of compressible flow. volume 27 of Oxford Lecture Series in Mathematics and its Applications. Oxford University Press, 2004.

[19] E. S. Oran and J. P. Boris. Numerical simulation of reactive flow. Cambridge University Press, 2001

[20] P. I. Plotnikov and Zh. Sokolovski. Stationary solutions of Navier-Stokes equations for diatomic gases. Uspekhi Mat. Nauk, 62(3(375)):117-148, 2007. 
I2M, Université D'Aix-Marseille, 39 Rue Joliot Curie, 13453 Marseille Cedx 13, France

E-mail address: afettah@cmi.univ-mrs.fr

I2M, Université D'Aix-Marseille, 39 Rue joliot Curie, 13453 Marseille Cedx 13, France

E-mail address: thierry.gallouet@univ-amu.fr

Université de Skikda, B.P.26 Route D'El-Hadaiek, 21000, Algérie

E-mail address: mer2iam@yahoo.fr 